\title{
Image Retrieval Techniques for Color based Images from Large Set of Database
}

\author{
R. Venkata Ramana \\ Chary \\ Padmasri Dr.B.V Raju Institute \\ of Technology, Hyderabad, \\ India
}

\author{
D. Rajya Lakshmi \\ GITAM Institute of Technology, \\ Visakhapatnam, India
}

\author{
K.V.N Sunitha \\ G. Narayanamma Institute of \\ Technology and Science \\ Hyderabad, India
}

\begin{abstract}
In content-based image retrieval, interactive systems have attracted a lot of research interest in recent years. Comparatively early systems, which focused on fully automatic strategies, recent approaches have supporting many are concentrated. Assuming that a user is looking for a set of images with similar feature using the query concept within a huge database Proposed system focus on the retrieval of images within a large image collection based on color projections and different geometric mathematical approaches are applied for retrieval of images . before Appling rules images are sub grouping using threshold method, in this paper R G B color combinations considered for retrieval of images and reduced comparing steps . this technique obtained fast and efficient results comparatively previous methods . the aim is to improve the efficiency to retrieve the query Concept.
\end{abstract}

Keywords: Color combination, threshold, Retrieval, Query, image Database

\section{INTRODUCTION}

Under computer vision techniques content based image retrieval $(\mathrm{CBIR})^{[2]}$ is one of the best method to retrieve the image set .Image retrieval is one of the interesting area to the image related problems. Automatic retrieval of images from a large collection database many methods are introduced from the long time .All methods are based on the colors, shapes and texture and image features ${ }^{[2]}$. All techniques used from fields such as pattern recognition ${ }^{[4][5]}$ and computer vision .

Content-Based Image Retrieval systems based on user-supplied bottom characteristics, directly find out images containing specific content from the image library. The basic process of the image processing is First of all we need to do appropriate preprocessing of images like resizing ,noise reduction and image transformation, and then extract image characteristics from the image based on the requirement all final contents of images to keep in the database for comparisons. When we retrieve to identify the image, extract the corresponding features ${ }^{[6][7]}$ from a known image and then retrieve the image database to identify the images which are similar with it, also we can give some of the characteristics based on a query requirement, then retrieve out the required images based on the given suitable values. In the whole retrieval process, feature extraction is essential; it is closely related to all aspects of the feature, such as color, shape, texture and space.

\subsection{Color Image}

A color image is basic colors composite of some basic true colors. In MATLAB programming image each pixel breaks into true color blocks of a color image .all values storing into 3 dimensional matrix into Red, Green and Blue values. we are going to get as a result, for the entire image is 3 matrices in 1.1 figure The three matrices are stacked next to each other creating a 3 dimensional $m$ by $n$ by 3 matrixes. an image which has a height of 5 pixels and width of 10 pixels the resulting in MATLAB would be a 5 by 10 by 3 matrixes for a true color image.

Figure 1 color Image and RGB matrix

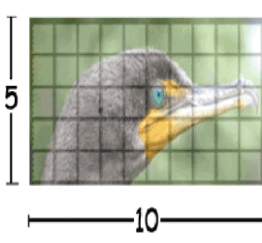

Original Color Image

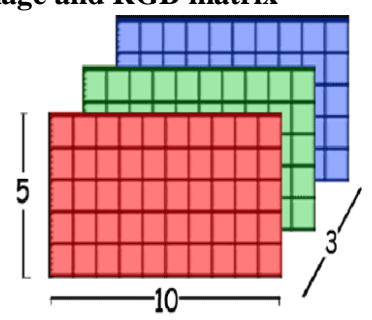

Matlab RCB Matrix

\subsection{Color Panel}

The figure 2 showing that color panels projecting the color components from the image through matrix R,G,B values we can project like

$$
\mathrm{R}=\mathrm{RGB}(:,:, 1) ; \mathrm{G}=\mathrm{RGB}(:,:, 2) ; \mathrm{B}=\mathrm{RGB}(:,:, 3) \text {;. }
$$

Figure 2 :RGB Color Panel
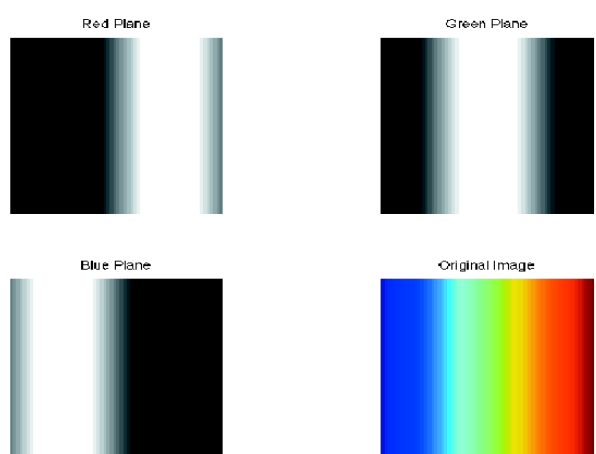


\subsection{Grayscale Image}

Figure 3: RGB color $s$ and related with Grayscale

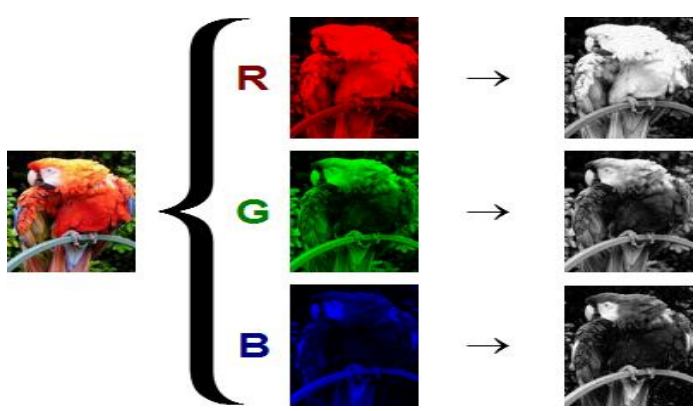

In grayscale ${ }^{[13]}$ model image colors are shades in gray color. In RGB color space red, green and blue components all have equal intensity values are projecting . for image in gray color it is only required to specify that a single intensity value for each pixel . Relation between $\mathrm{RGB}^{[14]}$ and Grayscale is presenting in the Figure 3: in this color channel splitting of a full RGB color image. In this picture The column at left shows the isolated color channels in natural colors, while at right there are their grayscale equivalences projections.

\subsection{Applications of Image Retrieval Systems :}

- System for Personal identification

- Employee recruitments

- Picture gallery

- List of catalogs

- therapeutic diagnosis

- Crime investigation

- military instruments

- Architectural design works
- $\quad$ engineering design

- GIS

- Forensic applications

\section{PROBLEM DESCRIPTION}

In image retrieval system for searching ${ }^{[3]}$, selecting and retrieving images from a large set of database of images various methods are following some of the methods are proposing adding metadata like keywords ${ }^{[1]}$,tokens, captioning , or descriptions to the images so that retrieval can be performed over the annotation words. so Manual image annotation is timeconsuming, laborious, expensive less performance to overcome this problem many researchers are proposed on automatic image retrievals using different methods .

Content-based means that the search will analyze the actual contents of the image. The term 'content' in this context might refer to colors, shapes, textures, or any other information that can be derived from the image itself.

\section{PROPOSED SYSTEM}

$>$ Proposed system uses combinations of color feature to overcomes the problem description.

$>$ Proposed system is implemented and concentrated on visual contents of an image such as color, shape, texture and spatial layouts.

$>$ Proposed system selected 10000 image database with feature values .

$>$ Proposed system extracted all images features separately R,G,B values for problem solving .

$>$ Proposed system implemented features like color histogram ,color projections

$>$ Mathematical approaches are proposed for efficient retrieval

$>$ Proposed work provides platform to extract images from the database using querying method 


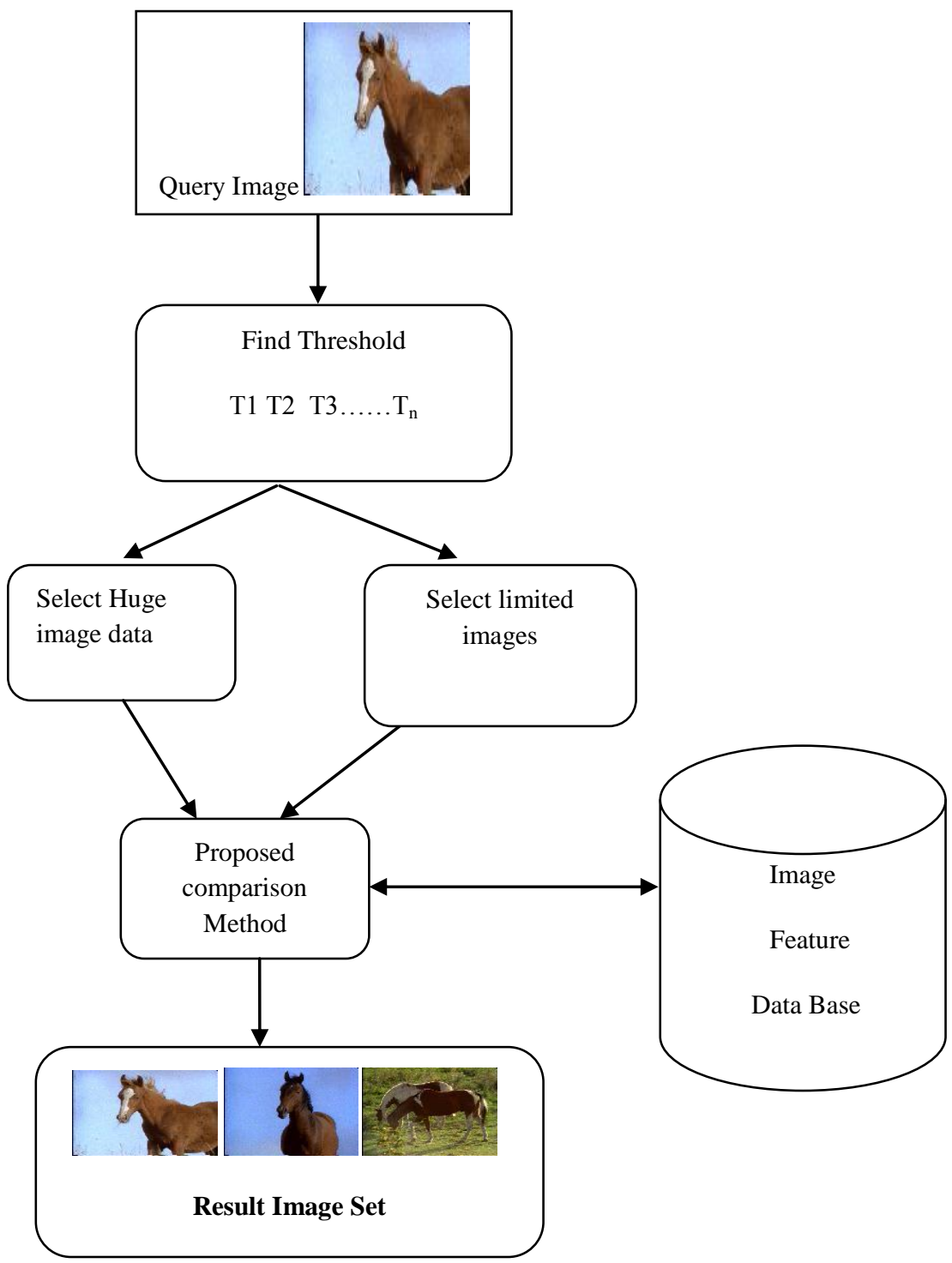

Figure 4 :Image Retrieval Svstem Architecture

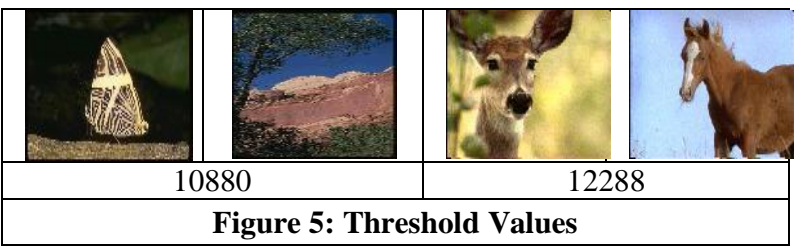

\section{RELATED WORK}

Image retrieval implemented in following steps :

Image retrieval is implemented in two phases one is new image insertion with features in to the database and other one is the new image searching in available database.

Step 1 :- All ten thousand images are taken in to working directory of MATLAB .

Step 2 :- Using MATLAB programming all images features calculated and stored into the database using specified programming methods.

Step 3:-Threshold calculation is taken for categorizing the images into the similar feature group s. in this step threshold value computed based on the histogram calculation.

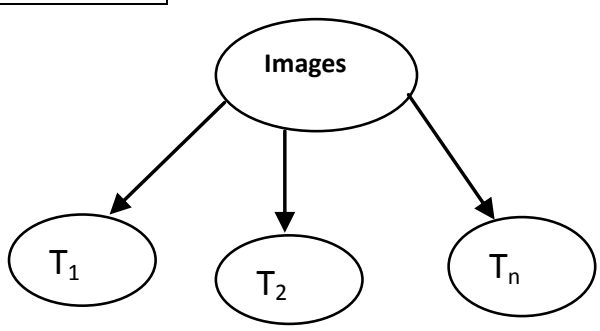

If the image is color image it is converting into gray color then calculated the sum of all bins in image histogram.

Figure 5: is one of the example with threshold values and images

If (isrgb(Image) )

GImage $=$ rgb2gray $($ Image $)$; threshold=sum(sum(p12))
p12=imhist(GImage); 
Using this method all images categorizing into $\mathrm{N}$ number Groups.

Step 4:In usual methods image color values storing in the matrix form . using Image matrix all this R , G, B components in image are extracted and separated into three different array forms ( Red, Green, Blue )

$$
\begin{aligned}
& \operatorname{Red}(\mathrm{M}, \mathrm{J})=\operatorname{Image}(\mathrm{M}, \mathrm{J}, 1) ; \\
& \operatorname{Green}(\mathrm{M}, \mathrm{J})=\operatorname{Image}(\mathrm{M}, \mathrm{J}, 2) ; \\
& \text { Blue }(\mathrm{M}, \mathrm{J})=\operatorname{Image}(\mathrm{M}, \mathrm{J}, 3) ;
\end{aligned}
$$

Step 5 : using the feature vectors each image color wise means are computed . in this method row and overall image mean is computed and stored into the database .based on all this features deferent computing methods are formulated.

\section{Mean_Row $=\operatorname{sum}($ Row Values $) /$ Number of Columns}

Mean_color=sum(Mean_Row) / Number of Rows

$$
\begin{aligned}
& \text { mean_r=mean2(Red); } \\
& \text { mean_g=mean2(Green); } \\
& \text { mean_b=mean2(Blue); }
\end{aligned}
$$

Step 6 :query image is selecting based on user choice and verifying threshold value if the threshold values is mapping the retrieval proposed methods are applying

Step 7 : using the image feature vectors various retrieval

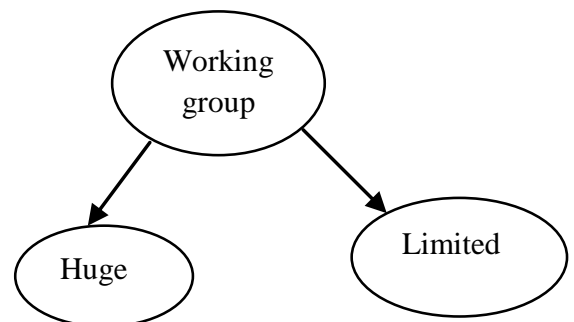

methods are proposed, in Each method two different working group sets are identifying one is Huge image set group and second one is limited set of image group

All Images $=\left\{T_{1}, T_{2}, T_{3} \ldots . . T_{n}\right\}$

Huge $=\mathrm{T}_{\text {equalent set }}\{\mathrm{T}$ is threshold value and all images belongs to same $\mathrm{T}$ group $\}$

Limit $=\mathrm{T}_{\text {equalent set } \text { with less images }}$

\section{Proposed Methods (PM) :-}

in each method two image feature values are verifying from available database files, after that comparing equalities of query image(QI) and target image(TI ). TI are may be similar or equal or not equal so based on the difference factor(DF) images are identifying .

$\mathrm{DF}=$ Constant $\{$ this value is proposed by user $\}$

PM 1 :- selecting the only one color mean value from image vector database means are comparing in the range of DF
PM 2 :- selecting the two colors mean values from the image feature database if the means are equal or similar values with in the range of DF .

Two color mean are like RG,RB,GB .

PM 3 :- selecting the three color mean values from the image feature database if the means are equal or similar values with in the range of $\mathrm{DF}$.

\section{Query Image:}
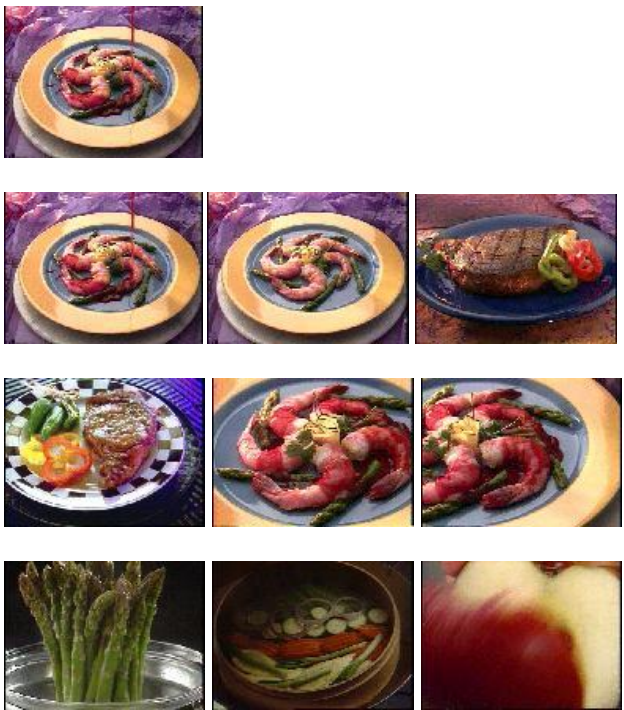

In this example one dish with plate is taken as a query image after that according to the method sequence image features are verified for this query red_mean value is taken 20 deferens factor is taken in the image set $66.6 \%$ successes rate is founded.

\subsection{Performance Measures}

\section{Precision}

Precision ${ }^{[13]}$ is the fraction of the images retrieved that are relevant to the user's information need.

precision $=\frac{\mid\{\text { relevent images }\} \cap\{\text { retrieved images }\} \mid}{\mid \text { retrieved images } \mid}$

Recall is the fraction of the images that are relevant to the query that are successfully retrieved.

recall $=\frac{\mid\{\text { relevent images }\} \cap\{\text { retrieved images }\} \mid}{\mid \text { relevant images } \mid}$ 


\section{EXPERIMENTAL RESULTS}

\begin{tabular}{|c|c|c|c|}
\hline \multicolumn{3}{|c|}{ Table 1: } \\
\hline p.name & R_mean & \multicolumn{2}{|c|}{ Picture } \\
\hline 998.jpg & 63.36859 & & \\
\hline 997.jpg & 65.08289 & & \\
\hline 995.jpg & 75.65936 & & \\
\hline 993.jpg & 82.29895 & & \\
\hline 992.jpg & 83.37225 & & \\
\hline
\end{tabular}

\begin{tabular}{|l|l|l|}
\hline 991.jpg & 84.91315 & \\
\hline 996.jpg & 86.83167 & \\
\hline 994.jpg & 110.7877 & \\
\hline
\end{tabular}

In table 1 it is showing that if the image is searching based on red_mean method Top 8 pictures are displaying in table mentioned order. In Table 1 : example DF value is 25 from picture 995.jpg. to remaining pictures.

\begin{tabular}{|l|r|r|r|r|r|l|l|r|}
\hline \multicolumn{8}{|c|}{ Table 2: Mean Feature values based on color } \\
\hline PIC & Threshold & R_MEAN & G & B & RG_AVG & RB & GB & RGB \\
\hline 991.jpg & 10880 & 84.91315 & 101.3946 & 70.87982 & 93.15388 & 51.93099 & 64.84949 & 85.72919 \\
\hline 992.jpg & 10880 & 83.37225 & 101.3106 & 93.328 & 92.34143 & 58.90008 & 64.55068 & 92.67028 \\
\hline 993.jpg & 10880 & 82.29895 & 104.5754 & 85.91925 & 93.43718 & 56.07273 & 66.00419 & 90.9312 \\
\hline 994.jpg & 10880 & 110.7877 & 107.5833 & 50.07007 & 109.1855 & 53.61926 & 72.25627 & 89.48036 \\
\hline 995.jpg & 10880 & 75.65936 & 77.5484 & 45.00427 & 76.60388 & 40.22121 & 51.38409 & 66.07068 \\
\hline 996.jpg & 10880 & 86.83167 & 93.98053 & 82.88165 & 90.4061 & 56.57111 & 61.46221 & 87.89795 \\
\hline 997.jpg & 10880 & 65.08289 & 74.93854 & 54.64471 & 70.01072 & 39.9092 & 48.31642 & 64.88871 \\
\hline 998.jpg & 10880 & 63.36859 & 76.91479 & 67.53912 & 70.14169 & 43.6359 & 49.01883 & 69.27417 \\
\hline
\end{tabular}

Table 3 : Image Retrieval Ranking List based on color

\begin{tabular}{|l|c|c|c|c|c|c|c|c|}
\hline \multicolumn{10}{|c|}{ Table 3 : Image Retrieval Ranking List based on color } \\
\hline PIC & TRISHOLD & R_MEAN & G & B & RG_AVG & RB & GB & RGB \\
\hline 991.jpg & 10880 & 6 & 6 & 5 & 6 & 4 & 6 & 4 \\
\hline 992.jpg & 10880 & 5 & 5 & 7 & 5 & 8 & 5 & 8 \\
\hline 993.jpg & 10880 & 4 & 7 & 8 & 7 & 6 & 7 & 7 \\
\hline 994.jpg & 10880 & 8 & 8 & 2 & 1 & 5 & 8 & 6 \\
\hline 995.jpg & 10880 & 3 & 3 & 1 & 3 & 2 & 3 & 2 \\
\hline 996.jpg & 10880 & 7 & 4 & 6 & 4 & 7 & 4 & 5 \\
\hline 997.jpg & 10880 & 2 & 1 & 3 & 1 & 1 & 1 & 1 \\
\hline 998.jpg & 10880 & 1 & 2 & 4 & 2 & 3 & 2 & 3 \\
\hline
\end{tabular}

The above Table 2 and Table 3 are showing that picture names ,threshold values and color means of R,G,B ,two color combinations and final RGB values calculated, those feature values are represented for top 8 images in table 2.based on table 2 feature values image retrieval ranks calculated and those values are represented in Table 3 .

From the feature values based on the color mean combination methods results are showing that almost 75 to 90 $\%$ of picture is looking similar. At the same time we can notify that the results for single color to combination of colors features if combining similar images are coming together this we can observe through ranking(1 to 8), in this observation we can conclude that instead of using single color projection we can select different combinations of color methods applying we can achieve good performance. 


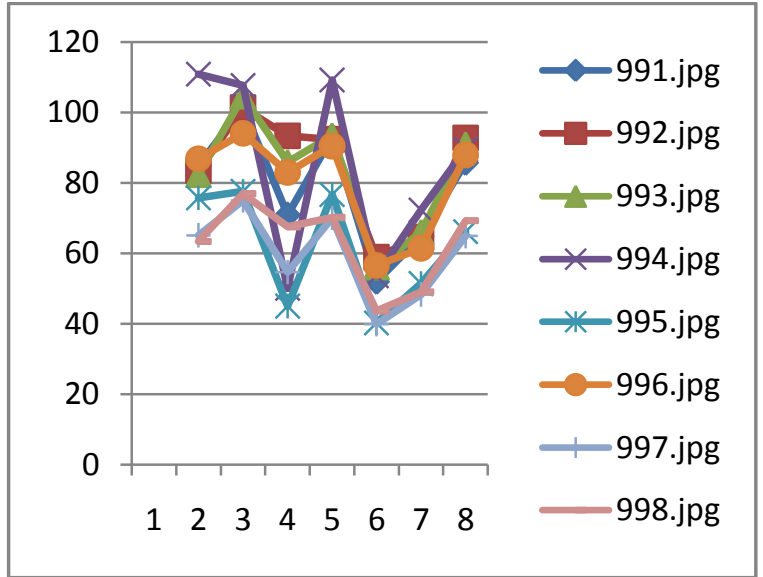

Figure 6 : R G B Mean Relation Graph

In Figure 6: Images graph showing that after Appling of color image mean rules ,relations in various methods is indicated .(2 is r_mean, 3 is g_mean,4 is b_mean,5 is rg_avg, 6is rb_avg,7is gb_avg, 8 is rgb_avg means.)

Second example : large image set taken as a input Deference factor is consider it as 20 result feature R G B values are showing in table 5 .

\begin{tabular}{|c|c|c|c|c|}
\hline \multicolumn{5}{|c|}{ Table 5: Image retrieval using $\mathrm{R}$ mean on flowers } \\
\hline Name & Picture & $\mathrm{R}$ mean & G mean & B mean \\
\hline 840.jpg & & 89.6731 & 77.64551 & 52.79016 \\
\hline 801.jpg & & 90.64164 & 67.11526 & 66.91756 \\
\hline 835.jpg & & 92.43567 & 92.7334 & 67.65942 \\
\hline 836.jpg & & 94.58813 & 86.33008 & 51.8114 \\
\hline 858.jpg & & 94.68475 & 97.16736 & 71.00061 \\
\hline 884.jpg & & 95.85382 & 99.8587 & 57.23804 \\
\hline 892.jpg & & 97.90094 & 80.11609 & 64.9588 \\
\hline 807.jpg & & 100.5533 & 67.95203 & 82.54828 \\
\hline 841.jpg & & 100.6967 & 78.23383 & 69.21942 \\
\hline 839.jpg & & 104.9514 & 114.3145 & 89.53088 \\
\hline 829.jpg & & 110.2375 & 61.48303 & 81.71808 \\
\hline
\end{tabular}
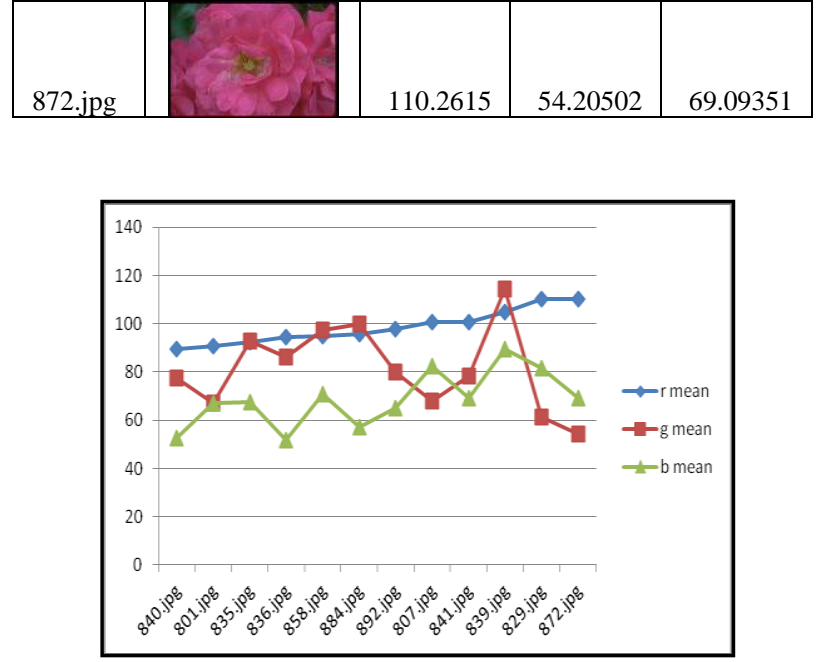

Figure 7 : Red Mean relation graph

In Figure 7 flower image set Red mean feature is projected for retrieval from image set .Red and other colors relations are represented in this graph .

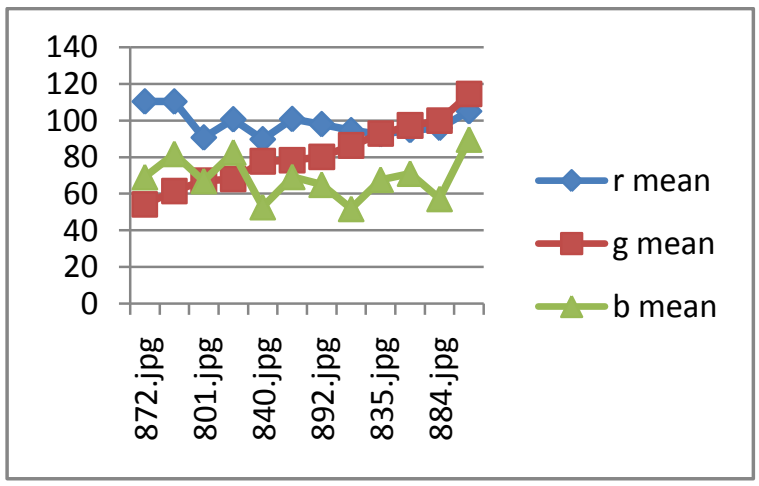

Figure 8 : Green mean relation graph

In Figure 8 flower image set green mean feature is projected for retrieval from image set. Green and other colors features relations are represented in this graph.

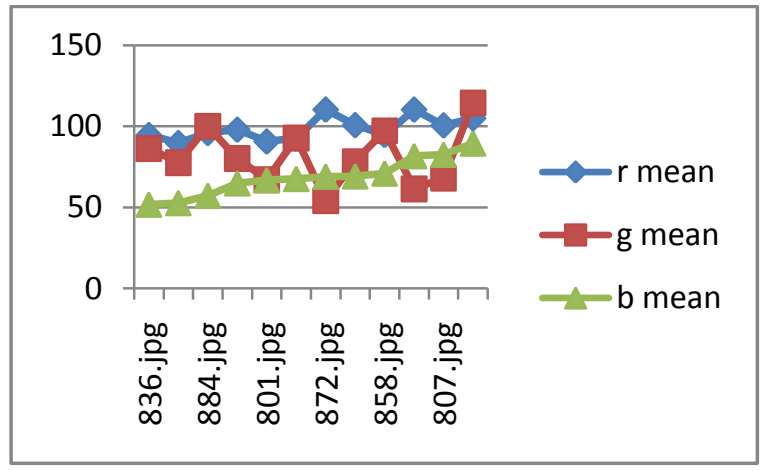

Figure 9 : Blue color mean order

In Figure 9 flower image set blue mean feature is projected for retrieval of image set blue and other colors features relations are represented in this graph. 


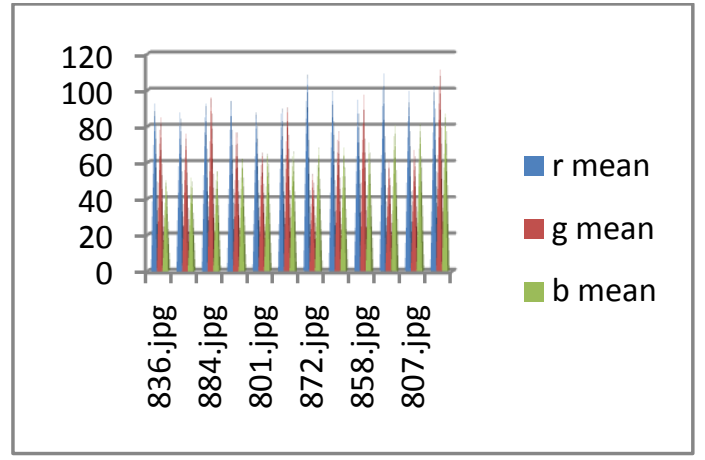

Figure 10 : Color projections relation graph

In Figure 10 flower image set mean feature is projected for retrieval of image all colors features relations are represented for each image using graphical representation.

\section{FUTURE ENHANCEMENT}

Most Internet-based Content-Based Image Retrieval (CBIR) systems focus on different photo collections and all systems not address challenges of large specialized image collections and topics such as semantic information retrieval by image content. In the research work we concentrated more than 10000 images with different categories of images and verified the image similar groups using threshold values calculations .present systems for grouping of images manual approaches are implemented in this work it is helping towards large set of image retrieval applications .

\section{CONCLUSION}

This method is efficient ${ }^{[13]}$ comparatively old methods which is used to retrieve the images from the huge database .in this work we compared total 10000 images with different categories . all suggested methods are helpful to perform the good results. Based on query images all retrieved images founded that vary similar. from method wise results are varying, best method is identified if we select the combinations of colors mean selections methods it gives the best performs . Current techniques are based on low level features and there is a huge semantic gap existing. in future days more research work is needed with user interest with a reliable and semantically competent system.

\section{REFERENCES}

[1] Image Annotation by Input-Output Structural Grouping Sparsity Han, Y.; Wu, F.; Tian, Q.; Zhuang, Y.; Y. Han is with the School of Computer Science and Technology, Tianjin University, China[JAN 2012]

[2] R.Datta, D.Joshi, J.Li, J.Z.Wang. "Image Retrieval: ideas, Influences, and Trends of the New Age". ACM Transactions on Com-puting Surveys, Vol. 40, No. 2(2008)

[3] Najlae Idrissi. "Bridging the Semantic Gap for Texture-based Image Retrieval and Navigation, Journal Of Multimedia", Vol.4, No.5, October 2009

[4] M. L. Kherfi, D. Ziou, and A. Benard. "Image retrieval from the World Wide Web: Issues, techniques and systems”. ACM Computing Surveys, 36(1):35\{67, 2006\}

[5] A. W. M. Smeulders , M. Worring, S. Santini, A. Gupta, and R. Jain. "Content-based image retrieval at the end of the early years". IEEE Transactions on Pattern Analysis and Machine Intelligence, 22(12):1349-1380, December 2008.

[6] Tag-based Image Retrieval Improved by Augmented Features and Group-based Refinement Chen, L.; Xu, D.; Tsang, I.; Luo, J.; IEEE(JAN 2012)

[7] P.L. Stanchev, D. Green Jr., B. Dimitrov, "High level color simi-larity retrieval", Int. J. Inf. Theories Appl. 10 (3) (2003) 363-369.

[8] V. Mezaris, I. Kompatsiaris, M.G. Strintzis, "An ontology approach to object-based image retrieval", Proceedings of the ICIP, vol. II, 2003, pp. 511-514 .

[9] Huan Wang, Song Liu, liang tien chia. "Image retrieval with a multi-modality ontology". Multimedia System (2008)

[10] Aigrain, P et al (1996) "Content-based representation and retrieval of visual media - a state-of-the-art review" Multimedia Tools and Applications 3(3), 179-202.

[11] Stricker, M and Orengo, M (1995) "Similarity of color images" in Storage and Retrieval for Image and Video Databases III (Niblack, W R and Jain, R C, eds), Proc SPIE 2420, pp 381-392

[12] Androutsas, D et al (1998) "Image retrieval using directional detail histograms" in Storage and Retrieval for Image and Video Databases VI, Proc SPIE 3312, 129-137

[13] R.Venkata Ramana Chary , Dr. D. Rajya Lakshmi, Dr. K.V.N. Sunitha Published an International Journal on "Image Retrieval and Similarity Measurement Based on Image Feature" in International Journal of Computer Science and Technology (IJCST) Volume 2 ,Issue 4 OCT-DEC 2011.PP385-389 ISSN :09768491(ONLINE)|ISSN:2229-4333(PRINT)

[14] R.Venkata Ramana Chary, Dr. K.V.N. Sunitha, Dr. D. Rajya Lakshmi Published an International journal "Quality Measures on Multispectral Images" in international journal of Image Processing and Applications(IJIPA) ,volume 2(1),2011,July- December 2011 .pp 101-105 ISSN:0975-8178.

\section{AUTHORS PROFILE}

R.Vekata Ramana Chary is working as a Associate professor In BVRIT College, Narsapur, Medak dist, AP. Presently he is pursuing PhD from GITAM University, AP, India. His research interest is in image processing, performance measurements, algorithms analysis and programming Techniques. He is having 15 years of teaching and research experience.

Dr. D. Rajya Lakshmi is working as Professor and HOD in the department of IT at GITAM University, Visakhapatnam, AP, India. Her research areas include Image processing and Data mining. She has about 17 years of teaching and research experience. Her PhD was awarded from JNTUH, Hyderabad, $\mathrm{AP}$ in the area of Image Processing.

Dr. K.V.N. Sunitha is working as a Professor and HOD in G. NARAYANAMMA INSTITUTE OF TECHNOLOGY AND SCIENCE (for WOMEN) in Hyderabad, AP. Her PhD was awarded from JNTUH Hyderabad AP. she is having 18 years of teaching and research experience in the computer field. Her research interest is in Compilers, NLP, Speech Recognition, Computer Networks and Image Processing. 